\title{
Electrophysiologic Studies and Radiofrequency Catheter Ablation of Ectopic Atrial Tachycardia in Children
}

\author{
Keiko Toyohara $\cdot$ Hitoo Fukuhara $\cdot$ Jun Yoshimoto $\cdot$ \\ Noriyasu Ozaki • Yoshihide Nakamura
}

Received: 11 July 2010/ Accepted: 18 September 2010/Published online: 10 October 2010

(C) The Author(s) 2010. This article is published with open access at Springerlink.com

\begin{abstract}
Ectopic atrial tachycardia (EAT) often resists medical therapy, making radiofrequency catheter ablation (RFCA) the preferred treatment. This study reviewed the records of 35 patients who underwent electrophysiologic studies (EPS) and 39 RFCA procedures for EAT during a 10 -year period. Of the 35 patients, $10(28 \%)$ presented with decreased ventricular function and tachycardia-induced cardiomyopathy (TIC). The EAT originated on the right atrial side in 19 patients $(54 \%)$ and on the left atrial side in the remaining 16 patients $(46 \%)$. The right atrial sites included the right atrial appendage (RAA) $(n=9,25 \%)$, the tricuspid annulus $(n=7,20 \%)$, and the crista terminalis $(n=3)$. The left atrial sites included the left atrial appendage (LAA) $(n=6,17 \%)$, the pulmonary veins $(n=5,14 \%)$, the mitral annulus $(n=3)$, and the posterior wall of the left atrium $(n=2)$. The mechanism of all EAT probably is automaticity. All EATs could be abolished using RFCA. Follow-up data were available for all patients 2 to 8 years after RFCA. All 35 patients remained recurrence free, and ventricular function improved for all 10 patients with TIC. The origin of EAT in children differed from its origin in adults. The authors conclude that RFCA is a safe and effective treatment option for children with refractory EAT and should be considered early in the course of their illness.
\end{abstract}

K. Toyohara $\cdot$ H. Fukuhara $\cdot$ J. Yoshimoto $\cdot$ N. Ozaki ·

Y. Nakamura

Japanese Red Cross Society, Wakayama Medical Center,

Wakayama, Japan

\section{K. Toyohara $(\bowtie)$}

Department of Pediatric Cardiology,

Tokyo Women's Medical University,

8-1 Kawada-cho, Shinjuku-ku, Tokyo 162-8666, Japan

e-mail: ktoyohar@hotmail.com
Keywords Catheter Ablation - Children . Ectopic atrial tachycardia

Ectopic atrial tachycardia (EAT) is a common chronic supraventricular tachycardia (SVT) in children $[3,5,16]$ that often resists medical therapy. Untreated, it may progress to tachycardia-induced cardiomyopathy (TIC) [4, 13], and differentiating it from dilated cardiomyopathy is sometimes difficult.

Radiofrequency catheter ablation (RFCA) of SVT in children has been successful, with a low risk of significant complications [1]. In adults, the foci of EAT often occur along the crista terminalis [8, 18] the tricuspid annulus [14], the ostium of the coronary sinus [9], the perinodal region [11], and the pulmonary veins [10]. This study aimed to assess the clinical presentation, natural history, and treatment response of EAT in children.

\section{Methods}

Patients

We retrospectively reviewed a consecutive series of 35 patients (17 girls and 18 boys; median age, 7 years; range 5 months to 15 years) with clinically documented paroxysmal or incessant EAT (Table 1). All the patients had clinically documented paroxysmal or incessant EAT. Patients with multiple changing atrial tachycardia morphologies or structural heart disease were excluded from the study. The age at onset of EAT varied from in utero to 14 years.

Two patients with paroxysmal EAT reported palpitations. The remaining 33 patients had no manifest symptoms. 


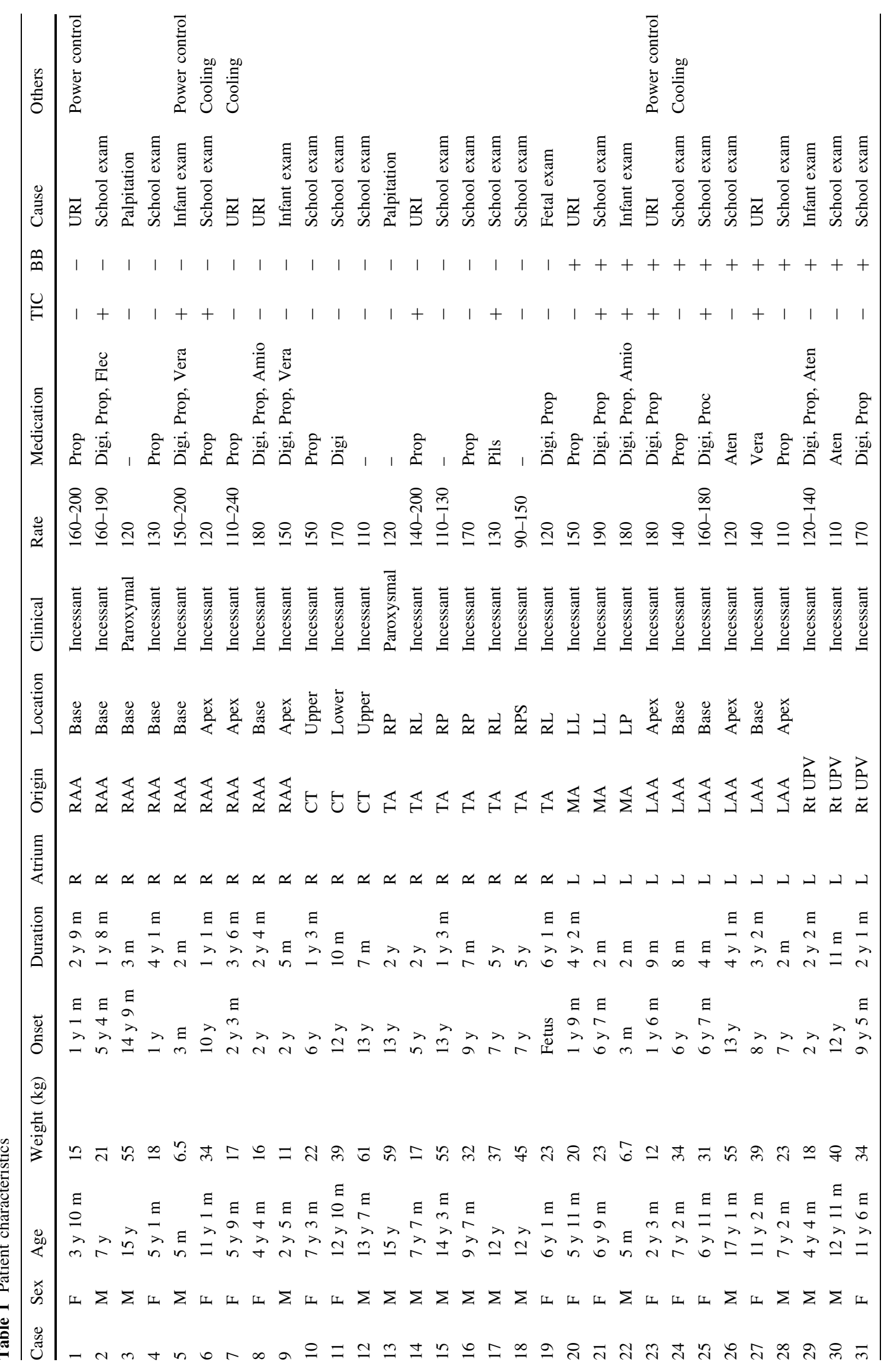




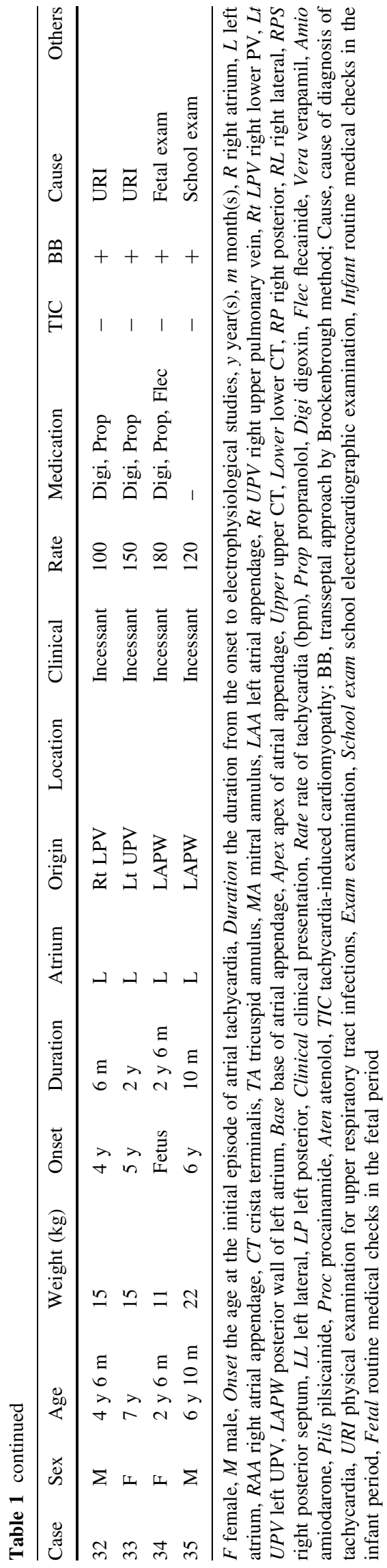

Incessant tachycardia was occasionally detected at school electrocardiographic (ECG) examination, at routine medical checks in the fetal or infant period, or during physical examination for upper respiratory tract infections. The 33 asymptomatic patients had incessant EAT. Of the 35 patients, $10(28 \%)$ already had decreased ventricular function and TIC when EAT was first diagnosed. The patients with decreased function had shortening fractions ranging from $20 \%$ to $27 \%$.

Antiarrhythmic medications were used as the initial therapy for 29 of the 35 patients, with drugs used for rate control, which was achieved for $25(86 \%)$ of the 29 patients although EAT persisted. Although rate was controlled for 6 of 10 patients with TIC, EAT, left ventricle dilatation, and decreased ventricular function persisted. Six patients or their parents chose participation in electrophysiologic studies (EPS) and RFCA in preference to drug treatment. Consequently, all 35 patients underwent EPS and RFCA. The mean duration from onset of EAT to the EPS was 2 years.

\section{Definition}

Ectopic atrial tachycardia is characterized by (1) atrial activation originating in a small area and spreading centrifugally; (2) a range of activation time less than the tachycardia cycle length, with the first few beats showing progressive shortening; (3) atrioventricular block possibly occurring in the presence of persisting SVT; (4) ventriculoatrial dissociation during ventricular pacing at the cycle length of the SVT without a change in the atrial activation sequence; and (5) typical inability to induce or terminate tachycardia with standard pacing protocols $[6,8,11,14$, 18]. Cardiomyopathy was defined as a shortening fraction of less than $28 \%$ or an ejection fraction of less than $45 \%$ $[4,12,13,15]$.

Tachycardias were considered to arise from the tricuspid or mitral annulus based on the following criteria [14]: (1) RFCA catheter positioned in an annular location when viewed in right and left anterior oblique fluoroscopic views with characteristic annular motion of the catheter tip and 2) an atrioventricular ratio less than 1 .

\section{EPS and RFCA}

All patients underwent EPS after providing informed written consent. We performed EPS and RFCA with the patient under general anesthesia using intubation and mechanical ventilation with blood pressure monitoring from the axillary artery. Two diagnostic 4-Fr catheters were positioned: one in the atrium and one in the coronary sinus. If possible, a 5-Fr catheter was placed at the His bundle electrogram site. A 7-Fr decapolar catheter (Biosense-Webster, Diamond 
Bar, CA, USA) was used for mapping via the inferior vena cava. We mapped during EAT using three-dimensional mapping for all 35 patients, and ablated at the earliest activation sites. We used the CARTO EP Navigation system (Biosense Webster) to obtain three-dimensional mapping during EAT in all 35 patients and ablated at the earliest activation sites. We did not use conventional mapping.

We usually used a temperature-controlled, 4-mm-tip ablating catheter (maximal temperature, $60^{\circ} \mathrm{C}$; maximal power, $30 \mathrm{~W}$ ) with titration of power and temperature. Acute procedural success was defined by the absence of tachycardia or ectopy $30 \mathrm{~min}$ after ablation despite infusion of isoproterenol and burst atrial pacing. When a leftsided origin was suspected, access to the left atrium for mapping and ablation was obtained transseptally using a Brockenbrough needle.

After the procedure, patients were treated with oral aspirin ( $3 \mathrm{mg} / \mathrm{kg} / \mathrm{day})$ for 1 month to prevent thromboembolic complications. Follow-up assessment was at 1, 3, 6, and 12 months, with clinical assessment and a 12-lead ECG. At 3 months, 24-h monitoring and echocardiography were performed.

\section{Statistical Analysis}

All continuous variables are expressed as mean \pm standard deviation. Comparisons between groups were performed with an unpaired Student $t$ test or a Mann-Whitney $U$ test. A $p$ value less than 0.05 was considered statistically significant. Detailed statistical analysis between different subgroups based on age or arrhythmia origin was limited because of the small sample sizes.

\section{Results}

Ectopic atrial tachycardia occurred spontaneously rather than in response to programmed extra stimulation or burst atrial pacing in any patient. After suppression by sedation, EAT was induced by isoproterenol infusion. A progressive increase in atrial rate with tachycardia onset (warm-up) or a progressive decrease before tachycardia termination (cooldown) was observed in all cases. We mapped during EAT and ablated at the earliest activation sites.

The distribution of EAT among the 35 patients was on the right atrial side in 19 patients (54\%) and on the left atrial side in 16 patients (46\%) (Fig. 1). The right atrial sites included the right atrial appendage (RAA) $(n=9$, $25 \%)$, the tricuspid annulus $(n=7,20 \%)$, and the crista terminalis $(n=3)$. The left atrial sites included the left atrial appendage (LAA) $(n=6,17 \%)$, the pulmonary veins $(n=5,14 \%)$, the mitral annulus $(n=3)$, and the posterior wall of the left atrium $(\mathrm{n}=2)$. We used a Brockenbrough

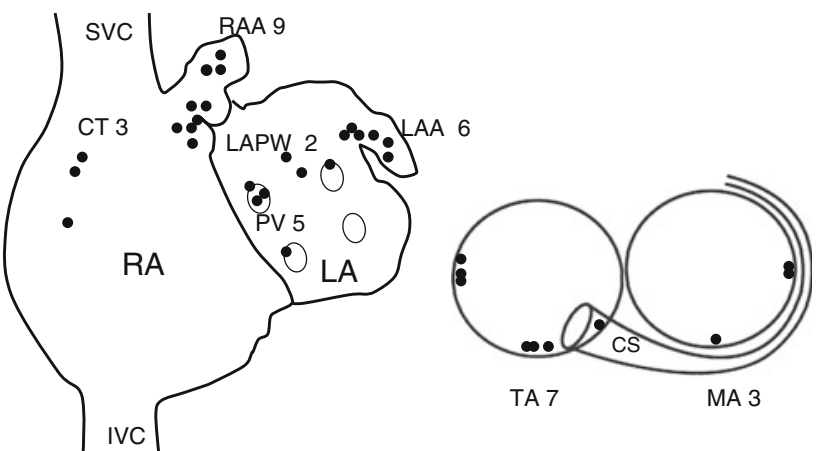

Fig. 1 Origin of atrial tachycardia. $S V C$ superior vena cava, $I V C$ inferior vena cava, $R A$ right atrium, $L A$ left atrium, $R A A$ right atrial appendage, $C T$ crista terminalis, $L A A$ left atrial appendage, $P V$ pulmonary vein, $L A P W$ posterior wall of left atrium, $T A$ tricuspid annulus, $M A$ mitral annulus

needle to enter the left atrium transseptally in 16 patients with EAT of left atrial origin. By contrast injection, we identified the anatomic location of the RAA and LAA. The origin of EAT was from the base of the triangulated component of the appendage in nine patients and from the tip of the appendage in six patients. There was no aneurysm of the atrial appendage.

We usually used a temperature-controlled ablating catheter with titration of power and temperature. In six cases with an EAT origin in the RAA or LAA, the output of radiofrequency (RF) could not be increased, so we changed to a power-controlled model (maximal temperature, $70^{\circ} \mathrm{C}$; maximal power, $20 \mathrm{~W}$ ). During $\mathrm{RF}$, we used an end-hole catheter irrigation to infuse saline near the RFCA target site, which enabled us to achieve sufficient power $(10-20 \mathrm{~W})$ for successful ablation. There were no episodes of perforation or thrombus.

In the short term, RFCA was successful for all 35 patients. Four patients had a recurrence of EAT within 1 month after RFCA. Two of the recurrences were located in the tricuspid annulus, one near the right upper pulmonary vein and one on the posterior wall of the left atrium. Successful repeat ablations were achieved for all patients who had a recurrence. Ultimately, we eliminated all EATs. There were no complications or recurrences over a mean follow-up period of $36 \pm 5$ months.

For 12 patients (34\%), the initial episode of SVT occurred before the age of 3 years (Table 2). In two cases, EAT was detected during a medical check-up at the age of 3 months. Both patients presented with decreased ventricular function. One patient had severe heart failure and needed cardiopulmonary bypass. The EAT was resistant to medical therapy, and rate control of the tachycardia proved difficult. For the remaining 10 patients, the duration of observation with drugs for rate control was from 5 months to 6 years, and EAT persisted during this time. 
Table 2 Clinical characteristics of each group divided by the period of initial episode of ectopic atrial tachycardia (EAT)

\begin{tabular}{|c|c|c|c|}
\hline \multicolumn{4}{|c|}{35 patients (M 18, F 17) } \\
\hline Onset & Fetus to $<3$ y 12 & 3 y to $<12$ y 16 & $12 \mathrm{y}$ to 7 \\
\hline \multirow[t]{2}{*}{ Sex } & M 4 & M 8 & M 6 \\
\hline & F 8 & F 8 & F 1 \\
\hline $\begin{array}{l}\text { Clinical } \\
\text { presentation }\end{array}$ & Incessant 12 & Incessant 16 & $\begin{array}{l}\text { Incessant } 5 \\
\text { Paroxysmal } 2\end{array}$ \\
\hline TIC & 3 & 7 & 0 \\
\hline \multirow[t]{7}{*}{ Site of EAT } & RAA 6 & RAA 2 & RAA 1 \\
\hline & TA 1 & TA 4 & TA 2 \\
\hline & MA 2 & MA 1 & \\
\hline & LAA 1 & LAA 4 & LAA 1 \\
\hline & PV 1 & PV 3 & PV 1 \\
\hline & LAPW 1 & LAPW 1 & \\
\hline & & CT 1 & CT 2 \\
\hline
\end{tabular}

TIC tachycardia-induced cardiomyopathy, $M$ male, $F$ female, $R A A$ right atrial appendage, $T A$ tricuspid annulus, $M A$ mitral annulus, $L A A$ left atrial appendage, $P V$ pulmonary vein, $L A P W$ posterior wall of left atrium, $C T$ crista terminalis

We performed RFCA in all 12 patients. Their age at the procedure varied from 5 months to 6 years. In 6 of the 12 cases, EAT originated in the RAA. In 6 of 9 cases of EAT originating from the RAA, the patients had their initial episode before the age of 3 years (Table 3 ).

Of the 35 patients, 10 (28\%) had already developed TIC when EAT was diagnosed. After RFCA, all 10 patients improved their shortening fraction from $22.8 \pm 5.1 \%$ to $38.7 \pm 11.7 \%(p<0.01)$, as measured by transthoracic echocardiography. The EAT causing TIC originated in the atrial appendage in six patients (RAA 3, LAA 3) and in the atrioventricular annulus in four patients (tricuspid annulus 2, mitral annulus 2).
Adenosine triphosphate (ATP) was administered during atrial tachycardias for 12 patients during EPS (mean dose, $5.2 \pm 2.4 \mathrm{mg}$; range, $3-15 \mathrm{mg} ; 0.1-0.5 \mathrm{mg} / \mathrm{kg}$ ). In all 12 cases, transient suppression occurred, but EAT recurred immediately.

\section{Discussion}

Ectopic atrial tachycardia is thought to caused by rapid discharges from an automatic atrial focus distinct from the sinus node. Histologic studies have shown that spontaneous activity arises from a single focus of abnormal cells. In adults, the foci of EAT most often occur along the crista terminalis $[8,18]$, the tricuspid annulus [14], the coronary sinus ostium [9], the perinodal region [11], and the pulmonary veins [10].

In contrast, our series of children showed that the ectopic focus more often is located in one of the atrial appendages. This difference in location of the ectopic focus suggests a possible difference in underlying substrate for EAT between children and adults. In younger children, the focus often may be a remnant of the original embryonic cells with abnormal automaticity. In this regard, the RAA originates from the primitive atrium, and the LAA is the remnant of the original embryonic left atrium that develops during the third week of gestation. We suggest that in children, persisting autorhythmic tissue may be the source of the arrhythmia. Of 12 patients younger than 3 years in our study, 6(50\%) had EAT originating from the RAA $(p<0.02)$.

As a focus for EAT, the crista terminalis is unlikely in children. The crista terminalis is an area of marked anisotropy due to poor transverse cell-to-cell coupling [15]. By creating a region of slow conduction, such anisotropy favors the development of microreentry. In addition, the

Table 3 Clinical characteristics of each group divided by the origin of ectopic atrial tachycardia (EAT)

\begin{tabular}{|c|c|c|c|c|c|c|c|}
\hline \multirow[t]{3}{*}{ Site of EAT } & \multicolumn{7}{|c|}{35 patients (M 18, F 17) } \\
\hline & RAA & $\mathrm{CT}$ & TA & MA & LAA & PV & LAPW \\
\hline & 9 & 3 & 7 & 3 & 6 & 5 & 2 \\
\hline \multirow[t]{2}{*}{ Sex } & M 4 & M 1 & M 6 & M 1 & M 2 & M 3 & M 1 \\
\hline & F 5 & F 2 & F 1 & F 2 & F 4 & F 2 & F 1 \\
\hline Clinical presentation & $\begin{array}{l}\text { Incessant } 8 \\
\text { Paroxysmal } 1\end{array}$ & Incessant 3 & $\begin{array}{l}\text { Incessant } 6 \\
\text { Paroxysmal } 1\end{array}$ & Incessant 3 & Incessant 6 & $\begin{array}{l}\text { Incessant } \\
5\end{array}$ & Incessant 2 \\
\hline TIC & 3 & 0 & 2 & 3 & 2 & 0 & 0 \\
\hline \multicolumn{8}{|l|}{ Onset } \\
\hline Fetus to $<3 \mathrm{y}$ & 6 & 0 & 1 & 2 & 1 & 1 & 1 \\
\hline $3 \mathrm{y}$ to $<12 \mathrm{y}$ & 2 & 1 & 4 & 1 & 4 & 3 & 1 \\
\hline $12 \mathrm{y}-$ & 1 & 2 & 2 & 0 & 1 & 1 & 0 \\
\hline
\end{tabular}

$R A A$ right atrial appendage, $C T$ crista terminalis, $T A$ tricuspid annulus, $M A$ mitral annulus, $L A A$ left atrial appendage, $P V$ pulmonary vein, $L A P W$ posterior wall of left atrium, $M$ male, $F$ female, $T I C$ tachycardia-induced cardiomyopathy 
normal sinus pacemaker complex is distributed along the long axis of the crista terminalis [2]. Hence, cells with spontaneous automaticity are to be found in this region. In our study, patients with crista terminalis-related EAT were older than 7 years, suggesting that the substrate of EAT originating in the crista terminalis might be acquired with age.

Adenosine-sensitive and microreentrant atrial tachycardias have been reported [7], with origins in the perinodal region and atrioventricular valve annulus in adults. The atrioventricular annulus (tricuspid annulus, mitral annulus) was the usual origin for EAT in our study, the mechanism being automaticity, not reentry. In 9 of 10 patients, EAT originated from a site in the free wall of the tricuspid annulus or mitral annulus (Fig. 1). The one exception arose from the tricuspid posterior septal region.

Warm-up and cool-down phenomena favor an automatic mechanism. Tachycardia could not be initiated or terminated with programmed electrical stimulation in any of our cases. Administration of ATP transiently suppressed EAT, but it quickly recurred. These findings support our view that the mechanism of EAT in our patients was most likely automaticity.

\section{RFCA}

Because of the lower flow at the tip than at the base of the RAA or LAA, we used a lower temperature and power at the tip. Irrigation RFCA appears to be an effective procedure for EAT of RAA [7] or LAA origin, but its use is not approved in our institution. As an alternative, we performed the RF procedure with a power-controlled model or infused saline during RF near the ablation site. Because the atrial appendage wall is very thin, perforation with cardiac tamponade is a significant risk, so catheter manipulation and ablation require great care. We were able to eliminate EAT originating from the atrial appendages during one session without complications.

Systolic dysfunction associated with chronic tachyarrhythmias is known as TIC [4, 13]. Of our 35 patients, 10 (28\%) presented with decreased ventricular function and TIC, an incidence similar to that reported in the literature $[3,5]$. Complication of EAT by TIC was more often observed in children than in adults. Medi et al. [13] reported a $10 \%$ incidence of TIC with EAT in adults. Children with EAT are less likely to be aware of palpitations and tachycardia episodes.

In our study, 33 of the 35 patients had no typical complaints when EAT was diagnosed, and their EAT was incessant. In the two patients who reported palpitations, the EAT was paroxysmal. With successful elimination of the tachycardia substrate, the patients experienced significant improvement in their left ventricular ejection fraction. This emphasizes the need to treat these patients aggressively to restore normal sinus rhythm and maintain ventricular function. Ectopic atrial tachycardia complicated by TIC commonly originates in the atrial appendages or atrioventricular annulus, and this association may reflect the incessant behavior of tachycardia originating in these locations.

A higher rate of spontaneous resolution in younger patients ( $<3$ years) compared with older patients ( $\geq 3$ years) has been reported [16]. In our study, 12 patients (34\%) had their initial episode of SVT before the age of 3 years. We performed RFCA in 12 cases. The age at the RFCA procedure varied from 5 months to 6 years. Spontaneous resolution of tachycardia was not uncommon in our study.

Although Blaufox et al. [1] supported the use of RFCA by experienced physicians for selected infants, the predominant view is that a favorable risk-to-benefit ratio may not exist for younger children. Additionally, there is concern regarding the late enlargement of RF lesions located in the immature atrial myocardium of younger children [17]. Although cryoablation appears to be an effective and safe procedure for children, its use is not approved in Japan, so it was unavailable for these patients.

All efforts should be made to treat younger children medically, at least through infancy. However, if they experience a cardiomyopathy, we recommend EPS with consideration of RFCA. In children, EAT is unlikely to resolve spontaneously, and antiarrhythmics are frequently ineffective. Because RFCA has been used successfully and safely, it should be considered early in the course of treatment, especially if there is an associated TIC.

\section{Conclusion}

The origin of EAT in children differed from that in adults. We conclude that RFCA is a safe and effective treatment option for children with refractory EAT and should be considered early in the course of their illness.

Open Access This article is distributed under the terms of the Creative Commons Attribution Noncommercial License which permits any noncommercial use, distribution, and reproduction in any medium, provided the original author(s) and source are credited.

\section{References}

1. Blaufox AD, Felix GL, Saul JP (2001) Radiofrequency catheter ablation registry. Radiofrequency catheter ablation in infants $\leq 18$ months old: when is it done and how do they fare? Shortterm data from the pediatric ablation registry. Circulation 104:2803-2808

2. Boineau JP, Canavan TE, Schuessler RB, Cain ME, Corr PB, Cox JL (1988) Demonstration of a widely distributed atrial pacemaker complex in the human heart. Circulation 77:1221-1237 
3. Cummings RM, William AT, Mahle A, Margaret J, Strieper A, Robert $M$ et al (2008) Outcomes following electroanatomic mapping and ablation for the treatment of ectopic atrial tachycardia in the pediatric population. Pediatr Cardiol 29:393-397

4. De Giovanni JV, Dindar A, Griffith MJ, Edgar Ra, Silove ED, Stumper O et al (1998) Recovery pattern of left ventricular dysfunction following ablation of incessant supraventricular tachycardia in infants and children. Heart 79:588-592

5. Dhala AA, Case CL, Gillette PC (1994) Evolving treatment strategies for managing atrial ectopic tachycardia in children. Am J Cardiol 74:283-286

6. Gillette PC, Garson AT (1977) Electrophysiologic and pharmacologic characteristics of automatic ectopic atrial tachycardia. Circulation 56:571-575

7. Iesaka Y, Takahashi A, Goya M, Soejima Y, Okamoto Y, Fujiwara $\mathrm{H}$ et al (1997) Adenosine-sensitive atrial reentrant tachycardia originating from the atrioventricular nodal transitional area. J Cardiovasc Electrophysiol 8:854-864

8. Kalman JM, Olgin JE, Karch MR, Hamdan M, Lee RJ, Lesh MD (1998) "Cristal tachycardias": origin of right atrial tachycardias from the crista terminalis identified by intracardiac echocardiography. J Am Coll Cardioi 31:451-459

9. Kistler PM, Fynn SP, Haqqani H, Stevenson H, Vohra JK, Morton JB et al (2005) Focal atrial tachycardia from the ostium of the coronary sinus: electrocardiographic and electrophysiological characterization and radiofrequency ablation. J Am Coll Cardiol 45:1488-1493

10. Kistler PM, Prashanthan S, Fynn S, Stevenson I, Hussin A, Vohra $\mathrm{J}$ et al (2003) Electrophysiological and electrocardiographic characteristics of focal atrial tachycardia originating from the pulmonary veins: acute and long-term outcomes of radiofrequency ablation. Circulation 108:1968-1975

11. Lai LP, Lin JL, Chen TF, Ko WC, Lien WP (1998) Clinical, electrophysiological characteristics, and radiofrequency catheter ablation of atrial tachycardia near the apex of Koch's triangle. PACE 21:367-374
12. Lang RM, Bierig M, Devereux RB, Flachskampf FA, Foster E, Pellikka PA et al (2005) Recommendations for chamber quantification: a report from the American Society of Echocardiography's Guidelines and Standards Committee and the Chamber Quantification Writing Group, developed in conjunction with the European Association of Echocardiography, a branch of the European Society of Cardiology. J Am Soc Echocardiogr 18:1440-1463

13. Medi C, Kalman JM, Haqqani H, Vohra JK, Morton JB, Sparks PB et al (2009) Tachycardia-mediated cardiomyopathy secondary to focal atrial tachycardia: long-term outcome after catheter ablation. J Am Coll Cardiol 53:1791-1797

14. Morton J, Sanders P, Das A, Vohra J, Sparks P, Kalman J (2001) Focal atrial tachycardia arising from the tricuspid annulus: electrophysiologic and electrocardiographic characteristics. J Cardiovasc Electrophysiol 12:653-659

15. Saffitz JE, Kanter HL, Green KG, Tolley TK, Beyer EC (1994) Tissue-specific determinants of anisotropic conduction velocity in canine atrial and ventricular myocardium. Circ Res 74:10651070

16. Salerno JC, Kertesz NJ, Friedman RA, Arnold L, Fenrich AL (2004) Clinical course of atrial ectopictachycardia is age-dependent: results and treatment in children $<3$ or $>$ or $=3$ years of age. JACC 43:438-444

17. Saul JP, Hulse JE, Papagiannis J, Van Praagh R, Walsh EP (1994) Late enlargement of radiofrequency lesions in infant lambs: implications for ablation procedures in small children. Circulation 90:492-499

18. Tang C, Scheinman M, Van Hare G, Epstein L, Fitzpatrick A, Lee $\mathrm{R}$ et al (1995) Use of P-wave configuration during atrial tachycardia to predict site of origin. J Am Coll Cardiol 26: $1315-1324$ 